\title{
Genome instability in blood cells of a BRCA1+ breast cancer family
}

\author{
Fengxia Xiao ${ }^{1 \dagger}$, Yeong C Kim ${ }^{1 \dagger}$, Carrie Snyder ${ }^{2}$, Hongxiu Wen ${ }^{1}$, Pei Xian Chen ${ }^{3}$, Jiangtao Luo ${ }^{4}$, Dina Becirovic ${ }^{2}$,
} Bradley Downs ${ }^{1}$, Kenneth $\mathrm{H}_{\text {Cowan }}{ }^{5}$, Henry Lynch ${ }^{2 *}$ and San Ming Wang ${ }^{1 *}$

\begin{abstract}
Background: BRCA1 plays an essential role in maintaining genome stability. Inherited BRCA1 germline mutation $(B R C A 1+)$ is a determined genetic predisposition leading to high risk of breast cancer. While BRCA1+ induces breast cancer by causing genome instability, most of the knowledge is known about somatic genome instability in breast cancer cells but not germline genome instability.
\end{abstract}

Methods: Using the exome-sequencing method, we analyzed the genomes of blood cells in a typical BRCA1+ breast cancer family with an exon 13-duplicated founder mutation, including six breast cancer-affected and two breast cancer unaffected members.

Results: We identified 23 deleterious mutations in the breast cancer-affected family members, which are absent in the unaffected members. Multiple mutations damaged functionally important and breast cancer-related genes, including transcriptional factor BPTF and FOXP1, ubiquitin ligase CUL4B, phosphorylase kinase PHKG2, and nuclear receptor activator SRA 1. Analysis of the mutations between the mothers and daughters shows that most mutations were germline mutation inherited from the ancestor(s) while only a few were somatic mutation generated de novo.

Conclusion: Our study indicates that BRCA1+ can cause genome instability with both germline and somatic mutations in non-breast cells.

Keywords: BRCA1+, Familial breast cancer, Exome sequencing, Bioinformatics, Germline mutation, Genome instability

\section{Background}

BRCA1 maintains genome stability through repairing double-strand DNA damage and other mechanisms [1]. The BRCA1 germline mutation $(B R C A 1+)$ is a wellknown genetic predisposition for inherited breast cancer [2-4]. Women who inherited BRCA1+ have a $60-80 \%$ risk of developing breast cancer by the age of 70 [5]. It is believed that $B R C A 1+$ leads to breast cancer by causing genome instability [6,7]. Indeed, many efforts have been made to determine the nature of $B R C A 1+$ induced genome instability. Cytogenetic studies in $B R C A 1+$ familial breast cancer showed the losses of $2 \mathrm{q}, 4 \mathrm{p}, 4 \mathrm{q}, 5 \mathrm{q}$, and $12 \mathrm{q}$ [8]; analysis of a breast cancer-derived cell line HCC1937 showed aneuploidy, loss of p53 and PTEN, and loss of

\footnotetext{
* Correspondence: htlynch@creighton.edu; sanming.wang@unmc.edu ${ }^{\dagger}$ Equal contributors

${ }^{2}$ Hereditary Cancer Center, Department of Preventive Medicine, Creighton University, Omaha, NE 68198, USA

'Department of Genetics, Cell Biology and Anatomy, Omaha, NE 68198, USA Full list of author information is available at the end of the article
}

heterozygosity ( $\mathrm{LOH}$ ) at multiple loci [9]; analysis in $B R C A 1+$ basal-like breast cancer identified the losses of the regions containing RB1, INPP4B, RAD17, RAD50, and $R A P 80$ [10], and large-scale chromosomal breakage, copy number loss and $\mathrm{LOH}[11,12]$. Of the three distinct classes of "simple", "amplifier" and "complex" DNA copynumber alterations defined for breast cancer, BRCA1+ breast cancer fits within the "complex" class [13]. Conditional Brca1 knockout in the mouse model results in breast tumor formation after a long latency, changes in the centrosomes, chromosomal gain and loss in specific segments orthologous to the genetic loci mutated in human breast cancer [14-16]. Data from these studies indicate that many types of genetic defects in the genome are caused by BRCA1+ induced genome instability.

A fundamental question remains to be answered, that is, whether $B R C A 1+$ could cause germline genome instability. Most BRCA1 mutations are founder mutations originated from the ancestor of the affected family passing 
through multiple generations. While $B R C A 1+$ is inherited at the beginning of fertilization of the BRCA1+ carriers, breast cancer will only develop in the reproductive age. Between fertilization and reproductive age, are there any genetic changes in BRCA1+ carrier genome besides the cancer-targeted breast cell genome, considering the essential roles of BRCA1 in maintaining genome stability? Nearly all genome instability studies in human breast cancer have been focused on breast cancer tissue where germline changes are considered as normal genome variations and disregarded. Therefore, the information derived from previous studies reflects mainly the somatic genome instability in breast cancer cells.

We hypothesize that the loss of BRCA1 function could cause genome instability in non-breast cancer cells. In this study, we used exome sequencing method to analyze the entire coding genes in the genomes of blood cells in a typical $B R C A 1+$ breast cancer family. Our study identified multiple recurrent germline and somatic mutations in the genomes of blood cells, highlighting that BRCA1+ can cause genome instability in both breast cancer cells and non-breast cancer cells.

\section{Methods}

The family used for the study

The breast cancer family used in this study contains a heterozygous founder mutation, a $6 \mathrm{~kb}$ frameshift duplication comprising exon 13 of BRCA1 (ins $6 \mathrm{~kb}$ exon13ter1460) that originated from northern British ancestors $[17,18]$. This mutation is regarded as one of the frequent founder mutations for BRCA1 mutation testing. Eight family members across two generations were selected for exome sequencing analysis. Of those, six females were diagnosed with breast cancer at the age of $62(\# 1)$, 53 (\#2), 35 (\#4), 35 (\#5), 36 (\#6), and 35 years old (\#7), and each had inherited the founder mutation. Two members were unaffected at the age of 65 (\#3, female) and 45 (\#8, male), neither inherited the mutation. \#9 and \#10 (fathers) were used to remove the variants transmitted to their daughters \#5, \#6 and \#7, accordingly (Figure 1). The use of the samples for the study was approved by the Institutional Review Boards of Creighton University and University of Nebraska Medical Center. All subjects signed the consent form to participate in cancer genetic study and to publish the details.

\section{Exome sequencing, mapping, variant calling and validation}

Genomic DNA from blood cells of the selected individuals was used for this study. Exome library preparation, capture, and sequencing were performed following the Illumina exome sequencing procedures. NimbleGen SeqCap EZ human exome V2.0 kit was used for exome capture. Paired-end sequences $(2 \times 100)$ were collected in the Illumina HiSeq2000 sequencer. The exome data were deposited in NCBI (Accession number SRR949927).

The exome sequences were mapped to the human genome reference sequence hg19 by Bowtie 2 using the default parameters in paired mode [19]. The resulting SAM files were converted to BAM files and the duplicates were
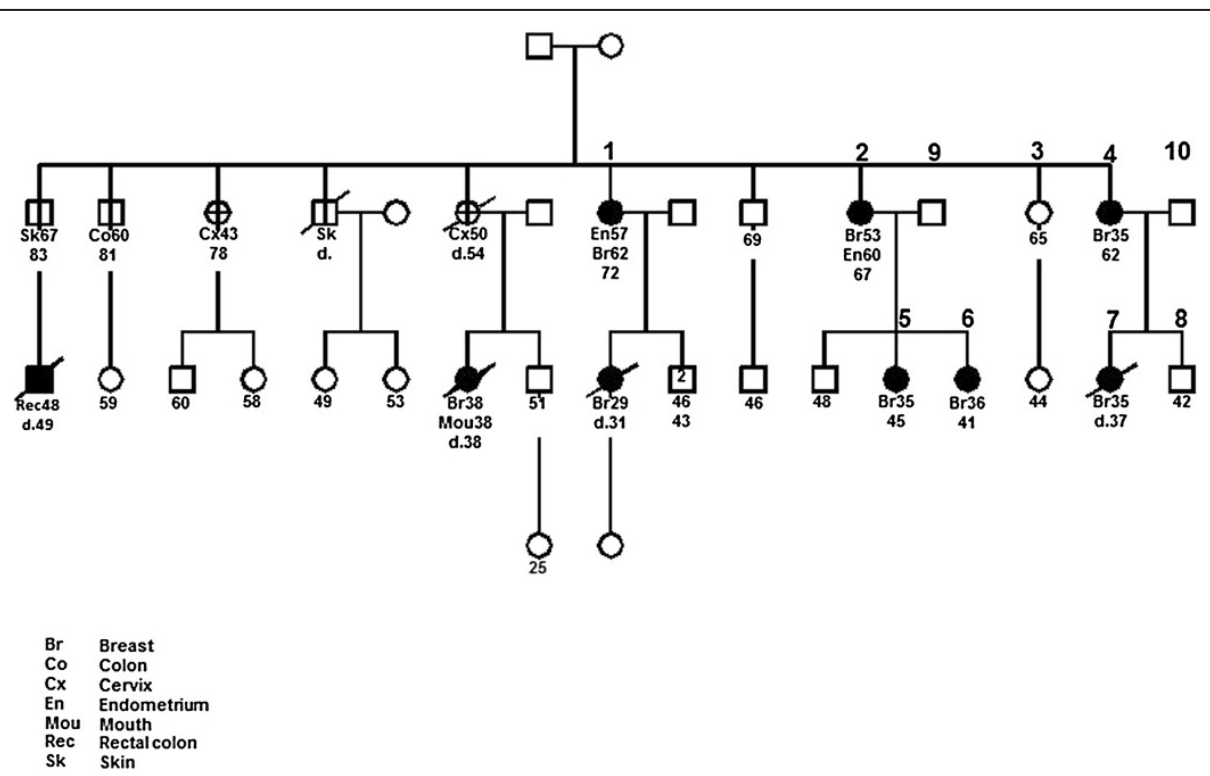

Figure 1 Pedigree of the BRCA1+ family used in the study. Eight members of this family were sequenced by exome sequencing, of which \#1 (62y), \#2 (53y), \#4 (35y), \#5 (35y), \#6 (36y), \#7 (35y) are breast cancer-affected and \#3 (65y) and \#8 (45y) are breast cancer-unaffected members. All six affected members are BRCA1+ whereas the two unaffected members are not. \#9 and \#10 were used in validation to remove the variants transmitted from them to their daughters. 
removed using Picard (http://picard.sourceforge.net). The mapped reads were locally realigned using GATK RealignerTargetCreator. The base quality scores were recalibrated with BaseRecalibrator using dbSNP137 in the GATK resource bundles for hg19.

VarScan 2 [20] and GATK [21] were used for variant calling following the instructions. For VarScan 2, pileup data were generated from BAM files using Samtools [22] mpileup command (with $-\mathrm{B}$ parameter to disable BAQ computation), and the default parameters were used with the minimum read depth at 10 , minimum base quality at 30; for GATK, UnifiedGenotyper was used for variant calling. BAM files were used for variant calling with GATK $\mathrm{v} 4$, release 2.0 with default parameter settings, including stand_call_conf $=30$ and stand_emit_conf $=30$, the minimum base quality score increased from 17 to 30 using dbSNP137. The variants called by VarScan 2 and GATK were annotated with ANNOVAR using the software provided databases of RefSeq, dbSNP137, 1000 Genomes [23] and ESP6500 from NHLBI Exome Sequencing Project (NHLBI GO Exome Sequencing Project, http://evs.gs. washington.edu/EVS/). The called variants were divided into known variants and novel variants. The known variants were further classified, based on their minor allele frequency (MAF) distribution in ESP6500 or 1000 Genome $(\leq 0.001$, and $>0.001)$. Those with MAF $>0.001$ were removed as common normal variants. Those with MAF $\leq$ 0.001 and novel variants were further classified into synonymous, nonsynonymous, splicing change, stop gain, and stop loss. For the nonsynonymous variants, PolyPhen2 [24] and SIFT [25] programs were used to identify those with predicted deleterious effects as defined by PolyPhen2 score [Probably damaging, 0.909-1, Possibly damaging 0.447 - 0.908, Benign 0-0.446 (HumVar score)], and SIFT score covered by ANNOVAR LJB2 (Damaging < 0.05 , Tolerant $\geq 0.05$ ) [26]. The final variants include the novel variants and the rare variants $(\mathrm{MAF} \leq 0.001)$ with deleterious effect, splicing alteration, and stop gain/loss. The fragile sites used for the analysis were based on the reference [27].

\section{Validation}

Sanger sequencing was used to validate the variants called by mapping analysis. Sense and antisense primers were designed for each candidate by Primer3 (http://frodo.wi. mit.edu/primer3/). PCR was performed with the same DNA used in exome sequencing (20 ng/reaction), sense and antisense primers (10 pmol), and Taq polymerase (1.25 unit, Promega) at the conditions of denaturing at $95^{\circ} \mathrm{C} 7$ minutes, 38 cycles at $95^{\circ} \mathrm{C} 30$ seconds, $56^{\circ} \mathrm{C}$ 30 seconds, $72^{\circ} \mathrm{C} 30$ seconds, final extension at $72^{\circ} \mathrm{C}$ 7 minutes. The amplified DNA products were subject to Big-Dye sequencing reactions. Sequences were collected in a ABI3730 sequencer, and examined by using
CLC Genomics Workbench 6.5 program (CLCbio, Cambridge, Massachusetts, USA) to validate the called variants.

\section{Results}

\section{Exome sequencing and variant calling}

We collected paired-end (2x100) exome sequences at 119x coverage on average for each member. We used the following steps for sequence mapping and variant call. 1) Sequences were processed and variants were called by both VarScan 2 and GATK; 2) The variants shared between the affected members and the unaffected members were removed; 3) Nonsynonymous variants were identified; 4) Deleterious variants leading to lossof-function of the affected genes were predicted by either PolyPhen-2 [24] or SIFT [25] or both programs. 5) The variants shared between the father $(\# 9, \# 10)$ and their daughters $(\# 5, \# 6, \# 7)$ were removed upon Sanger sequencing validation; 6) All the remaining variants were validated by Sanger sequencing to confirm that each variant is a real germline mutation present only in the breast cancer-affected members.

Through these processes, we identified 23 germline mutations in breast cancer-affected members in this family, of which 21 are novel mutations and 2 are rare mutations (rs143160739, rs370052455) with minor allele frequency (MAF) $<0.001$ (Table 1). The total frequency of the 23 mutations is 54 , including 12 (52\%) mutations present between 2 to 6 members, and 11 (48\%) mutations present only in a single individual. On average, 9.2 mutations $(54 / 6)$ are present in each breast cancer-affected member.

\section{Distribution of the mutations in the family}

Of the 23 mutations, 12 were shared in at least two of the three $B R C A 1+$ breast cancer-affected sisters (\#1, \#2, \#4), 2 were present only in sister \#2 (Table 2A). We compared the variants between mother and daughter pairs. Pair 1 includes mother (\#2) and two daughters (\#5, \#6), all are $B R C A 1+$ and affected with breast cancer at 53, 36 and 36 years old respectively; Pair 2 includes mother (\#4) and

Table 1 Exome data and variant calls

\begin{tabular}{cccc}
\hline Case & Total bases & Coverage & Variant called \\
\hline 1 & $5,333,230,980$ & 133 & 46,317 \\
2 & $3,715,176,060$ & 93 & 43,510 \\
3 & $8,558,235,540$ & 213 & 50,533 \\
4 & $5,621,290,920$ & 140 & 47,064 \\
5 & $3,915,557,820$ & 98 & 45,531 \\
6 & $3,209,882,580$ & 80 & 40,598 \\
7 & $3,605,428,440$ & 90 & 40,211 \\
8 & $4,143,350,070$ & 103 & 43,996 \\
Average & $4,762,769,051$ & 119 & 44,720 \\
\hline
\end{tabular}


Table 2 Germline mutations identified in the family

\begin{tabular}{|c|c|c|c|c|c|c|c|c|c|}
\hline \multirow[t]{2}{*}{ Gene } & \multirow[t]{2}{*}{ Position } & \multirow[t]{2}{*}{ Base change } & \multirow[t]{2}{*}{ AA change } & \multirow[t]{2}{*}{ Type } & \multicolumn{2}{|c|}{ SIFT } & \multicolumn{2}{|c|}{ PolyPhen 2} & \multirow[t]{2}{*}{ Frequency } \\
\hline & & & & & Score & Prediction & Score & Prediction & \\
\hline SRA1 & chr5:139936828 & c.C91T & p.P31s & SNV & 0.00 & D & 1.00 & D & 6 \\
\hline PHKG2* & chr16:30767746 & c.C706T & p.R236W & SNV & 0.00 & D & 1.00 & $\mathrm{D}$ & 5 \\
\hline ZNF24 & chr18:32919897 & c.T464G & p.M155R & SNV & 0.02 & $\mathrm{D}$ & 0.00 & $\mathrm{~B}$ & 4 \\
\hline TMPRSS7 & chr3:111797705 & c.G1963A & p.G655S & SNV & 0.00 & D & 1.00 & $\mathrm{D}$ & 4 \\
\hline ABLIM2 & chr4:8055946 & c.G791A & p.R264Q & SNV & 0.05 & D & 1.00 & $\mathrm{D}$ & 3 \\
\hline FOXP1 & chr3:71247489 & c.C44T & p.A15V & SNV & 0.34 & $\mathrm{~T}$ & 0.98 & D & 3 \\
\hline GSTK1 & chr7:142964824 & c.G703A & p.G235R & SNV & 0.02 & D & 1.00 & $\mathrm{D}$ & 3 \\
\hline LACRT & chr12:55028594 & c.C32T & p.A11V & SNV & 0.00 & D & 0.99 & $\mathrm{D}$ & 3 \\
\hline PAMR1 & chr11:35456266 & c.G1420A & p.G474R & SNV & 0.00 & D & 1.00 & D & 3 \\
\hline TTN & chr2:179400887 & c.G73392A & p.W24464X & Stop gain & NA & NA & NA & NA & 3 \\
\hline UEVLD & chr11:18553971 & c.G1312T & p.V438L & SNV & 0.37 & $\mathrm{~T}$ & 0.96 & $\mathrm{D}$ & 2 \\
\hline ITGA1 & chr5:52240783 & c.C3296G & p.S1099C & SNV & 0.01 & $\mathrm{D}$ & 1.00 & D & 2 \\
\hline BPTF & chr17:65850386 & c.A944C & p.N315T & SNV & 0.00 & D & 1.00 & $\mathrm{D}$ & 1 \\
\hline CACNB3 & chr12:49220218 & c.G688A & p.A230T & SNV & 0.01 & D & 1.00 & $D$ & 1 \\
\hline CUL4B & chrX:119680410 & c.A838T & p.R280X & Stop gain & NA & NA & NA & NA & 1 \\
\hline SEMA3C & chr7:80374250 & c.G2216A & p.R739Q & SNV & 0.01 & D & 0.99 & $\mathrm{D}$ & 1 \\
\hline TBC1D22B & chr6:37280778 & c.G1067A & p.S356N & SNV & 0.03 & D & 0.39 & $\mathrm{~B}$ & 1 \\
\hline CHCHD1 & chr10:75541868 & c.G35T & p.R12L & SNV & 0.01 & $\mathrm{D}$ & 0.99 & D & 1 \\
\hline KCTD8* & chr4:44177010 & c.C1219T & p.R407C & SNV & 0.00 & $\mathrm{D}$ & 1.00 & D & 1 \\
\hline CLCN4 & chrX:10176455 & c.G1214A & p.C405Y & SNV & 0.00 & D & 0.98 & $\mathrm{D}$ & 1 \\
\hline LAT & chr16:29000901 & c.G634T & p.A212S & SNV & 0.02 & D & 1.00 & $\mathrm{D}$ & 1 \\
\hline ZNF304 & chr19:57868409 & C.A1172T & p.Y391F & SNV & 0.16 & $\mathrm{~T}$ & 1.00 & D & 1 \\
\hline ZNF674 & chrX:46359537 & c.C1487A & p.P496H & SNV & 0.00 & $\mathrm{D}$ & 1.00 & D & 1 \\
\hline
\end{tabular}

*Two mutations exist in ESP 6500 with MAF 0.000077, all others are novel mutations only detected in this family.

$\mathrm{D}$ : probably damaging; P: possibly damaging; B: benign; T: tolerant.

daughter (\#7), both are BRCA1+ and were affected with breast cancer at 35 years old. Because the variants shared between the father and the daughter(s) were eliminated, each mutation is firmly determined as either germline mutation inherited from the mother, or somatic mutation generated de novo in the daughter(s). Of the 23 mutations, 15 were germline mutations between the mother and the daughter(s) (9 in Pair 1, and 6 in Pair 2); 9 were de novo somatic mutation only in the daughter (\#5). There was no de novo somatic mutation in the daughter (\#6) in Pair 1 or the daughter (\#7) in Pair 2 (Table 2B).

\section{Chromosomal distribution and ratio of transition/ transversion of the mutations}

The 23 mutations are enriched in several chromosomes, such as chromosome 3 (TMPRSS7, FOXP1), chromosome 16 (LAT, PHKG2) and chromosome X (CLCN4, CUL4B, ZNF674). Except the mutations in ZNF304 and $Z N F 674$, no mutations are located in the repetitive sequences of SINE, LINE, LTR, simple or satellite sequences. Fifteen mutations are located in the regions with known structural variations and 5 mutations in the chromosomal fragile sites of 4A, 11E, 2G, 11C, and 18A (Table 3). For the 23 mutations, the ratio of transition/transversion (Ti/Tv) is 1.6. However, the ratio increased to $3.0(9 / 3)$ for the common mutations. In contrast, the ratio decreased to $0.8(5 / 6)$ for the mutations present only in single individual. The ratio between common mutations and individual mutations is statistically different by Fisher exact test $(\mathrm{p}=0.009$, Table 4$)$.

\section{Functional categories of the mutated genes}

Multiple mutations have deleterious effects on functinal importance and breast cancer-related genes (Table 5, Additional file 1: Table S1). Followings are examples of the mutation-affected genes:

BPTF is a bromodomain PHD finger transcription factor, involved in transcriptional regulation and chromatin remodeling [28]. Copy number changes in BPTF are present in many types of cancer [29].

CUL4B is an E3 ubiquitin ligase catalysing polyubiquitination for protein degradation [30]. 
Table 3 Mutation distribution in the family

\begin{tabular}{|c|c|c|c|c|c|c|c|}
\hline Gene & Position & Base change & AA change & $\begin{array}{c}\text { Sister \#1 } \\
\text { Cancer }\end{array}$ & $\begin{array}{c}\text { Sister \#2 } \\
\text { Cancer }\end{array}$ & $\begin{array}{c}\text { Sister \#4 } \\
\text { Cancer }\end{array}$ & $\begin{array}{c}\text { Sister \#3 } \\
\text { Unaffected }\end{array}$ \\
\hline \multicolumn{8}{|c|}{ A. Mutation distribution in the three breast cancer-affected sisters } \\
\hline PHKG2 & chr16:30767746 & c.C706T & p.R236W & + & + & + & - \\
\hline GSTK1 & chr7:142964824 & c.G703A & p.G235R & + & + & - & - \\
\hline ITGA1 & chr5:52240783 & c.C3296G & p.S1099C & + & + & - & - \\
\hline PAMR1 & chr11:35456266 & c.G1420A & p.G474R & + & + & - & - \\
\hline TMPRSS7 & chr3:111797705 & C.G1963A & p.G655S & + & + & - & - \\
\hline UEVLD & chr11:18553971 & c.G1312T & p.V438L & + & + & - & - \\
\hline LACRT & chr12:55028594 & c.C32T & p.A11V & + & - & + & - \\
\hline SRA1 & chr5:139936828 & c.C91T & p.P31S & + & - & + & - \\
\hline ZNF24 & chr18:32919897 & c.T464G & p.M155R & + & - & + & - \\
\hline ABLIM2 & chr4:8055946 & c.G791A & p.R264Q & - & + & + & - \\
\hline FOXP1 & chr3:71247489 & c.C44T & p.A15V & - & + & + & - \\
\hline TTN & chr2:179400887 & c.G73392A & p.W24464X & - & + & + & - \\
\hline CACNB3 & chr12:49220218 & c.G688A & p.A230T & - & + & - & - \\
\hline $\mathrm{CHCHD1}$ & chr10:75541868 & c.G35T & p.R12L & - & + & - & - \\
\hline \multicolumn{8}{|c|}{ B. Mutation distribution between generations } \\
\hline Pair 1 & & & & Father \#9 & Mother \#2 & Daughter \#5 & Daughter \#6 \\
\hline RAB3C & chr5:58147140 & c.G646A & p.E216K & - & + & + & + \\
\hline SRA1 & chr5:139936828 & c.C91T & p.P31s & - & + & + & + \\
\hline TMPRSS7 & chr3:111797705 & c.G1963A & p.G655S & - & + & + & + \\
\hline ZNF24 & chr18:32919897 & c.T464G & p.M155R & - & + & + & + \\
\hline LACRT & chr12:55028594 & c.C32T & p.A11V & - & + & + & - \\
\hline PAMR1 & chr11:35456266 & c.G1420A & p.G474R & - & + & + & - \\
\hline GSTK1 & chr7:142964824 & c.G703A & p.G235R & - & + & - & + \\
\hline PHKG2 & chr16:30767746 & c.C706T & p.R236W & - & + & - & + \\
\hline UEVLD & chr11:18553971 & c.G1312T & p.V438L & - & + & - & + \\
\hline ITGA1 & chr5:52240783 & c.C3296G & p.S1099C & - & + & - & - \\
\hline BPTF & chr17:65850386 & C.A944C & p.N315T & - & - & + & - \\
\hline TBC1D22B & chr6:37280778 & c.G1067A & p.S356N & - & - & + & - \\
\hline SEMA3C & chr7:80374250 & c.G2216A & p.R739Q & - & - & + & - \\
\hline CUL4B & chrX:1 19680410 & c.A838T & p.R280X & - & - & + & - \\
\hline CLCN4 & chrX:10176455 & c.G1214A & p.C405Y & - & - & + & - \\
\hline LAT & chr16:29000901 & c.G634T & p.A212S & - & - & + & - \\
\hline ZNF304 & chr19:57868409 & c.A1172T & p.Y391F & - & - & + & - \\
\hline ZNF674 & chrX:46359537 & c.C1487A & p.P496H & - & - & + & - \\
\hline KCTD8 & chr4:44177010 & c.C1219T & p.R407C & - & - & + & - \\
\hline Pair 2 & & & & Father \#10 & Mother \#4 & Daughter \#7 & Son \#8 \\
\hline SRA1 & chr5:139936828 & c.C91T & p.P31S & - & + & + & + \\
\hline PHKG2 & chr16:30767746 & c.C706T & p.R236W & - & + & + & - \\
\hline CASQ1 & chr1:160165804 & c.G769A & p.E257K & - & + & + & - \\
\hline ABLIM2 & chr4:8055946 & c.G791A & p.R264Q & - & + & + & - \\
\hline TTN & chr2:179400887 & c.G73392A & p.W24464X & - & + & + & - \\
\hline FOXP1 & chr3:71247489 & c.C44T & p.A15V & - & + & + & - \\
\hline
\end{tabular}


Table 4 Genomic features of the mutations

\begin{tabular}{|c|c|c|c|c|c|c|}
\hline \multicolumn{7}{|c|}{ A. Genome distribution of the mutations } \\
\hline Gene & Position & Repetitive sequence & Structural variation & & Fragile $s$ & \\
\hline BPTF & chr17:65850386 & - & + & - & - & - \\
\hline CACNB3 & chr12:492220218 & - & + & - & - & - \\
\hline CHCHD1 & chr10:75541868 & - & + & - & - & - \\
\hline FOXP1 & chr3:71247489 & - & + & - & - & - \\
\hline GSTK1 & chr7:142964824 & - & + & - & - & - \\
\hline ITGA1 & chr5:52240783 & - & + & - & - & - \\
\hline LACRT & chr12:55028594 & - & + & - & - & - \\
\hline PHKG2 & chr16:30767746 & - & + & - & - & - \\
\hline SRA1 & chr5:139936828 & - & + & - & - & - \\
\hline CLCN4 & chrX:10176455 & - & + & - & - & - \\
\hline LAT & chr16:29000901 & - & + & - & - & - \\
\hline KCTD8 & chr4:44177010 & - & + & - & - & - \\
\hline ABLIM2 & chr4:8055946 & - & + & $4 \mathrm{~A}$ & 5149099 & 8732840 \\
\hline PAMR1 & chr11:35456266 & - & + & $11 \mathrm{E}$ & 31043424 & 36443424 \\
\hline TTN & chr2:179400887 & - & + & $2 \mathrm{G}$ & 169791754 & 182991755 \\
\hline UEVLD & chr11:18553971 & - & - & $11 C$ & 16143424 & 21643424 \\
\hline ZNF24 & chr18:32919897 & - & - & $18 \mathrm{~A}$ & 32746002 & 37246002 \\
\hline CUL4B & chrX:119680410 & - & - & - & - & - \\
\hline SEMA3C & chr7:80374250 & - & - & - & - & - \\
\hline TBC1D22B & chr6:37280778 & - & - & - & - & - \\
\hline TMPRSS7 & chr3:111797705 & - & - & - & - & - \\
\hline ZNF304 & chr19:57868409 & + & - & - & - & - \\
\hline ZNF674 & chrX:46359537 & + & - & - & - & - \\
\hline
\end{tabular}

B. Transition/ transversion of the mutations

\begin{tabular}{cccc}
\hline Wild type & Mutation & Wild type & Mutation \\
\hline Common mutations $(>=2)$ & & & \\
C & $T$ & $T$ & G \\
C & $T$ & $G$ & T \\
C & T & & G \\
C & $T$ & & \\
G & $T$ & & \\
G & A & & \\
G & A & \\
$G$ & A &
\end{tabular}

Individual mutations

$C$
$G$
$G$
$G$

$\begin{array}{ll}A & T \\ A & T \\ A & C \\ G & T \\ G & T \\ C & T \\ & \text { A }\end{array}$


Table 4 Genomic features of the mutations (Continued)

\begin{tabular}{cccc}
\hline C. Transition/Transversion ratio & & \\
\hline \multicolumn{1}{c}{ Class } & Transition & Transversion & Ratio \\
\hline Total mutation & 14 & 9 & 1.6 \\
Common & 9 & 3 & 3 \\
Individual & 5 & 6 & 0.8 \\
\hline
\end{tabular}

FOXP1 is a member of the forkhead box (FOX) transcription factor family, involving in regulation of tissueand cell type-specific gene expression. Its expression is under the regulation of estrogen and it is known to play a role in breast cancer cell proliferation [31].

GSTK1 (glutathione S-transferase kappa 1) is involved in cellular detoxification [32].

LACRT (lacritin) is highly expressed in lacrimal glands. Copy number amplification of LACRT was observed in breast cancer [33].

PHKG2 is a phosphorylase kinase. It is involved in liver glycogenesis. Mutations in this gene cause glycogen storage disease type $9 \mathrm{C}[34]$.

Table 5 Function of mutation-damaged genes

\begin{tabular}{|c|c|c|}
\hline Genes & Function & Mutation type \\
\hline ABLIM2 & Actin binding & nonsynonymous SNV \\
\hline BPTF & Transcriptional regulation & nonsynonymous SNV \\
\hline CACNB3 & Calcium channel & nonsynonymous SNV \\
\hline CHCHD1 & Nuclear protein & nonsynonymous SNV \\
\hline CLCN4 & Chloride channel & nonsynonymous SNV \\
\hline CUL4B & Polyubiquitination & stop gain \\
\hline FOXP1 & Transcriptional regulation & nonsynonymous SNV \\
\hline GSTK1 & Cellular detoxification & nonsynonymous SNV \\
\hline ITGA1 & Cell-cell adhesion & nonsynonymous SNV \\
\hline KCTD8 & Potassium channel & nonsynonymous SNV \\
\hline LACRT & Lacrimal gland development & nonsynonymous SNV \\
\hline LAT & $\begin{array}{l}\text { TCR - and pre-TCR-mediated } \\
\text { signaling }\end{array}$ & nonsynonymous SNV \\
\hline PAMR1 & Muscle regeneration & nonsynonymous SNV \\
\hline PHKG2 & $\begin{array}{l}\text { Phosphorylase kinase for } \\
\text { glycogenesis }\end{array}$ & nonsynonymous SNV \\
\hline SEMA3C & Developmental regulation & nonsynonymous SNV \\
\hline SRA1 & $\begin{array}{l}\text { Nuclear and non-nuclear } \\
\text { receptor Regulation }\end{array}$ & nonsynonymous SNV \\
\hline TBC1D22B & Unknown & nonsynonymous SNV \\
\hline TMPRSS7 & $\begin{array}{l}\text { Serine protease for peptide } \\
\text { hydrolyzes }\end{array}$ & nonsynonymous SNV \\
\hline TTN & Structural protein for chromosomes & stop gain \\
\hline UEVLD & Unknown & nonsynonymous SNV \\
\hline ZNF24 & Transcriptional regulation & nonsynonymous SNV \\
\hline ZNF304 & Transcriptional regulation & nonsynonymous SNV \\
\hline ZNF674 & Transcriptional regulation & nonsynonymous SNV \\
\hline
\end{tabular}

SRA1 is a steroid receptor activator, involving in the regulation of many nuclear and non-nuclear receptors and associating with breast cancer [35]. The mutation in this gene is also present in the member (\#8), who is a male not affected with breast cancer.

\section{Comparison between germline mutations and somatic mutations in breast cancer}

The Cancer Genome Atlas Network (TCGA) identified 56 somatically mutated genes from 510 breast cancer tissues, of which about $90 \%$ are sporadic breast cancer [36]. Comparison between the 23 mutated genes in our study and the 56 mutated genes shows no overlap between the two sets of mutation-affected genes. The closest correlation is the mutations in the FOX family, in which FOXA1 is somatically mutated in sporadic breast cancer and FOXP1 is germline-mutated in the BRCA1+ family. Mutations in these two $F O X$ genes are associated with breast cancer [37]. Absence of overlapping mutations between the $B R C A 1+$ genomes and sporadic breast cancer genomes suggests the different genetic basis between these two types of breast cancer. Searching the 23 mutations by gene name in the COSMIC database, which contain somatic mutation information for various types of human cancer, shows nineteen genes being present in COSMIC, however the mutations were at different position; searching by exact positions shows the presence of the same mutations for KCTD 8 and CACNB3. KCTD8 is a component of potassium channel and CACNB3 is $a$ subunit of calcium channel. The oncological roles of these two mutated genes in familial breast cancer remain to be elucidated.

\section{Discussion}

Tumorigenesis requires multiple genetic defects to transform a normal cell to a tumor cell $[38,39]$. In familial breast cancer, germline mutations inherited from ancestors play important roles in cancer processes. BRCA1+ is the strongest germline predisposition for familial breast cancer. While it is widely accepted that $B R C A 1+$ leads to breast cancer by causing genome instability, the detailed mechanism for how BRCA1+ causes genome instability remain to be determined. Our study shows the presence of mutations in blood cells from the BRCA1+ breast cancer family. By definition, only the mutations occurred shortly after fertilization can be germline mutation. 
Although blood cells are frequently used in solid tumor genetic study to represent germline genome, the mutations detected in blood cells in fact include both true germline mutations occurred during fertilization and somatic mutations occurred after fertilization. By combining the pedigree information, however, we can clearly distinguish the two types of mutations that those shared between generations (mothers and daughters) are the germline mutation, and those only presented in single individual (\#2 daughter) are de novo somatic mutations. The number of germline mutations is much larger than the number of de novo somatic mutations, this is due to the fact that germline mutations are accumulated/inherited from multiple generations whereas somatic mutations are only present in individual generation. The results indicate that BRCA1+ can induce germline genome instability represented by the genome of blood cells.

Can the small numbers of mutations be identified by chance? For the following reasons, we consider it unlikely: in the study, we applied a multi-step mapping pipeline in order to maximally differentiate germline mutations associated with cancer from abundant normal variants, including the filtration of normal variants using public variant databases of dbSNP, 1000 genomes and exome variation databases, the use of the aged, unaffected family members to remove private variants in the family, exclusion of the contribution of father's germline mutation to the daughter in the trios, the focus only on the deleterious mutations causing non-synonymous mutations, splicing alternation and stop gain/loss, and the use of Sanger sequencing validation. It is also worth indicating that the functionally important mutations in cancer are in small numbers $[40,41]$. The small number of mutations identified were likely generated by $B R C A 1+$ directly or indirectly. Their roles could be promoting oncogenesis, or function as $B R C A 1+$ modifiers to amplify the oncogenic function of $B R C A 1+$. Our study focuses on the deleterious mutations at single-based level. Other types of genetic changes could also be present in BRCA1+ genome. For example, increased CNV in TP53 was shown to be present in Li-Fraumeni syndrome, a disease closely related with BRCA1+ familial breast cancer [42].

It is well known that transition occurs at a higher rate than transversion mutations in cancer cells [40]. Indeed, we observed a higher $\mathrm{Ti} / \mathrm{Tv}$ ratio (3.0) than that in the normal genome (2.1) for the common mutations, implying that the germline genome instability also follows the same trend that found in somatic genome mutation. However, the ratio of $\mathrm{Ti} / \mathrm{Tv}$ mutations in the individual mutations $(0.8)$ is lower than that in the normal genome. Possible causes could be that the individual mutations tend to be random events, with less biological significance, or that the number of those mutations is too small for the comparison.
Elimination of family-specific normal variants is a key to identify the true predispositive mutations in the cancer, as a normal human genome can have multiple genes mutated [43]. Familial-specific normal variants cannot be removed solely by referring to the population-based variation databases, as many familial-specific normal variants are not included. The unaffected family members can serve as the closest control for this purpose. While this process could remove certain real mutations shared between the unaffected and affected members (low penentrant), and certain family-specific normal variants could still remain in the affected members (not present in the unaffected members), the mutations present only in the affected members are more likely to be associated with cancer than those shared between the affected and unaffected members. Referring to functional importance of the mutation-affected genes also helps to identify ture predispositive mutations. Because of the mutations we identified are present only in the cancer members, shared between multiple cancer-affected members in the disease family, and have functional relevance to cancer, they are most likely associated with breast cancer in the disease family.

\section{Conclusions}

Our study shows the presence of genome instability in the genomes of non-breast cells in the BRCA1+ familial breast cancer family. The presence of germline mutations provides a potential source to identify genetic targets for early intervention of tumorogenesis process in $B R C A 1+$ carriers long before tumor formation in breast cells, for which there are currently limited options besides preventive surgery.

\section{Availability of supporting data}

The exome data generated by the sutdy were deposited in NCBI (Accession number SRP028652). url: http://www. ncbi.nlm.nih.gov/Traces/sra/?study=SRP028652

\section{Additional file}

Additional file 1: Table S1. Validation by Sanger sequencing.

Competing interests

The authors declare that they have no competing interests.

\section{Authors' contributions}

FX, HW, PXC, BD performed experiments, YK performed bioinformatic data analysis, CS, DB identified the subjects and prepared DNA samples, JL performed statistical analysis, KY. HL, SMW conceived the study, SMW designed the experiment and wrote the paper. All authors read and approved the final manuscript.

\section{Acknowledgments}

The study was supported by a pilot grant from Fred \& Pamela Buffett Cancer Center, University of Nebraska Medical Center (SMW), a NIH grant 1R21CA180008 (SMW), an Overseas Study Scholarship by China Scholarship 
Council (FX). the Charles F. and Mary C. Heider Chair in Cancer Research (HL) and by revenue from Nebraska cigarette taxes awarded to Creighton University by the Nebraska Department of Health and Human Services (HL).

\section{Author details}

'Department of Genetics, Cell Biology and Anatomy, Omaha, NE 68198, USA. ${ }^{2}$ Hereditary Cancer Center, Department of Preventive Medicine, Creighton University, Omaha, NE 68198, USA. ${ }^{3}$ Department of Medicine, College of Medicine, Omaha, NE 68178, USA. ${ }^{4}$ Department of Biostatistics, College of Public Health, Omaha, NE 68178, USA. ${ }^{5}$ Fred \& Pamela Buffett Cancer Center, University of Nebraska Medical Center, Omaha, NE 68178, USA.

Received: 17 January 2014 Accepted: 7 May 2014

Published: 19 May 2014

\section{References}

1. Welcsh PL, King MC: BRCA1 and BRCA2 and the genetics of breast and ovarian cancer. Hum Mol Genet 2001, 10:705-713.

2. Hall JM, Lee MK, Newman B, Morrow JE, Anderson LA, Huey B, King MC: Linkage of early-onset familial breast cancer to chromosome 17q21. Science 1990, 250:1684-1689.

3. Narod SA, Feunteun J, Lynch HT, Watson P, Conway T, Lynch J, Lenoir GM: Familial breast-ovarian cancer locus on chromosome 17q12-q23. Lancet 1991, 338:82-83.

4. Miki Y, Swensen J, Shattuck-Eidens D, Futreal PA, Harshman K, Tavtigian S, Liu Q, Cochran C, Bennett LM, Ding W, Bell R, Rosenthal J, Hussey C, Tran T, McClure M, Frye C, Hattier T, Phelps R, Haugen-Strano A, Katcher H, Yakumo K, Gholami Z, Shaffer D, Stone S, Bayer S, Wray C, Bogden R, Dayananth P, Ward J, Tonin P, Narod S: A strong candidate for the breast and ovarian cancer susceptibility gene BRCA1. Science 1994, 266:66-71.

5. U.S. Preventive Services Task Force: Genetic risk assessment and BRCA mutation testing for breast and ovarian cancer susceptibility: recommendation statement. Ann Intern Med 2005, 143:355-361.

6. Venkitaraman AR: Cancer susceptibility and the functions of BRCA1 and BRCA2. Cell 2002, 108:171-182.

7. Stratton MR, Rahman N: The emerging landscape of breast cancer susceptibility. Nat Genet 2008, 40:17-22.

8. Tirkkonen M, Johannsson O, Agnarsson BA, Olsson H, Ingvarsson S, Karhu R, Tanner M, Isola J, Barkardottir RB, Borg A, Kallioniemi OP: Distinct somatic genetic changes associated with tumor progression in carriers of BRCA1 and BRCA2 germ-line mutations. Cancer Res 1997, 57:1222-1227.

9. Tomlinson GE, Chen TT, Stastny VA, Virmani AK, Spillman MA, Tonk V, Blum JL, Schneider NR, Wistuba II, Shay JW, Minna JD, Gazdar AF: Characterization of a breast cancer cell line derived from a germ-line BRCA1 mutation carrier. Cancer Res 1998, 58:3237-3242.

10. Weigman VJ, Chao HH, Shabalin AA, He X, Parker JS, Nordgard SH, Grushko T, Huo D, Nwachukwu C, Nobel A, Kristensen VN, Børresen-Dale AL, Olopade OI, Perou CM: Basal-like Breast cancer DNA copy number losses identify genes involved in genomic instability, response to therapy, and patient survival. Breast Cancer Res Treat 2012, 133:865-880.

11. Popova T, Manié E, Rieunier G, Caux-Moncoutier V, Tirapo C, Dubois T, Delattre O, Sigal-Zafrani B, Bollet M, Longy M, Houdayer C, Sastre-Garau X, Vincent-Salomon A, Stoppa-Lyonnet D, Stern M: Ploidy and large-scale genomic instability consistently identify basal-like breast carcinomas with BRCA1/2 inactivation. Cancer Res 2012, 72:5454-5462.

12. Konishi H, Mohseni M, Tamaki A, Garay JP, Croessmann S, Karnan S, Ota A, Wong HY, Konishi Y, Karakas B, Tahir K, Abukhdeir AM, Gustin JP, Cidado J Wang GM, Cosgrove D, Cochran R, Jelovac D, Higgins MJ, Arena S, Hawkins L, Lauring J, Gross AL, Heaphy CM, Hosokawa Y, Gabrielson E, Meeker AK, Visvanathan K, Argani P, Bachman KE, Park BH: Mutation of a single allele of the cancer susceptibility gene BRCA1 leads to genomic instability in human breast epithelial cells. Proc Natl Acad Sci U S A 2011, 108:17773-17778.

13. Kwei KA, Kung Y, Salari K, Holcomb IN, Pollack JR: Genomic instability in breast cancer: pathogenesis and clinical implications. Mol Oncol 2010, 4:255-266.

14. Xu X, Wagner KU, Larson D, Weaver Z, Li C, Ried T, Hennighausen L, Wynshaw-Boris A, Deng CX: Conditional mutation of Brca1 in mammary epithelial cells results in blunted ductal morphogenesis and tumour formation. Nat Genet 1999, 22:37-43.
15. Shen $S X$, Weaver Z, Xu X, Li C, Weinstein M, Chen L, Guan XY, Ried T, Deng CX: A targeted disruption of the murine Brca1 gene causes gamma-irradiation hypersensitivity and genetic instability. Oncogene 1998, 17:3115-3124.

16. Brodie SG, Xu X, Qiao W, Li WM, Cao L, Deng CX: Multiple genetic changes are associated with mammary tumorigenesis in Brca1 conditional knockout mice. Oncogene 2001, 20:7514-7523.

17. Puget N, Sinilnikova OM, Stoppa-Lyonnet D, Audoynaud C, Pagès S, Lynch HT, Goldgar D, Lenoir GM, Mazoyer S: An Alu-mediated 6-kb duplication in the BRCA1 gene: a new founder mutation? Am J Hum Genet 1999, 64:300-302.

18. The BRCA1 Exon 13 Duplication Screening Group: The exon 13 duplication in the BRCA1 gene is a founder mutation present in geographically diverse populations. Am J Hum Genet 2000, 67:207-212.

19. Langmead B, Salzberg SL: Fast gapped-read alignment with Bowtie2. Nat Methods 2012, 9:357-359.

20. Koboldt DC, Zhang Q, Larson DE, Shen D, McLellan MD, Lin L, Miller CA, Mardis ER, Ding L, Wilson RK: VarScan 2: somatic mutation and copy number alteration discovery in cancer by exome sequencing. Genome Res 2012, 22:568-576.

21. McKenna A, Hanna M, Banks E, Sivachenko A, Cibulskis K, Kernytsky A, Garimella K, Altshuler D, Gabriel S, Daly M, DePristo MA: The genome analysis toolkit: a MapReduce framework for analyzing next-generation DNA sequencing data. Genome Res 2010, 20:1297-1303.

22. Li H, Handsaker B, Wysoker A, Fennell T, Ruan J, Homer N, Marth G, Abecasis G, Durbin R, 1000 Genome Project Data: The sequence alignment/map (SAM) format and SAMtools. Bioinformatics 2009, 25:2078-2079.

23. The 1000 Genomes Project Consortium: An integrated map of genetic variation from 1,092 human genomes. Nature 2012, 491:56-65.

24. Adzhubei IA, Schmidt S, Peshkin L, Ramensky VE, Gerasimova A, Bork P, Kondrashov AS, Sunyaev SR: A method and server for predicting damaging missense mutations. Nat Methods 2010, 7:248-249.

25. Kumar $\mathrm{P}$, Henikoff $S, \mathrm{Ng} P \mathrm{PC}$ : Predicting the effects of coding non-synonymous variants on protein function using the SIFT algorithm. Nat Protoc 2009, 4:1073-1081.

26. Wang $K, L i M$, Hakonarson H: ANNOVAR: Functional annotation of genetic variants from next-generation sequencing data. Nucleic Acids Res 2010, 38:e164

27. Fungtammasan A, Walsh E, Chiaromonte F, Eckert KA, Makova KD: A genome-wide analysis of common fragile sites: what features determine chromosomal instability in the human genome? Genome Res 2012, 22:993-1005.

28. Ruthenburg AJ, Li H, Milne TA, Dewell S, McGinty RK, Yuen M, Ueberheide B, Dou Y, Muir TW, Patel DJ, Allis CD: Recognition of a mononucleosomal histone modification pattern by BPTF via multivalent interactions. Cell 2011, 145:692-706.

29. Buganim Y, Goldstein I, Lipson D, Milyavsky M, Polak-Charcon S, Mardoukh C, Solomon H, Kalo E, Madar S, Brosh R, Perelman M, Navon R, Goldfinger N, Barshack I, Yakhini Z, Rotter V: A novel translocation breakpoint within the BPTF gene is associated with a pre-malignant phenotype. PLoS One 2010, 5:e9657.

30. Nakagawa T, Xiong Y: X-linked mental retardation gene CUL4B targets ubiquitylation of $\mathrm{H} 3 \mathrm{~K} 4$ methyltransferase component WDR5 and regulates neuronal gene expression. Mol Cell 2011, 43:381-391.

31. Shigekawa T, ljichi N, Ikeda K, Horie-Inoue K, Shimizu C, Saji S, Aogi K, Tsuda H Osaki A, Saeki T, Inoue S: FOXP1, an estrogen-inducible transcription factor, modulates cell proliferation in breast cancer cells and 5-year recurrence-free survival of patients with tamoxifen-treated breast cancer. Horm Cancer 2011, 2:286-297.

32. Robinson A, Huttley GA, Booth HS, Board PG: Modelling and bioinformatics studies of the human Kappa-class glutathione transferase predict a novel third glutathione transferase family with similarity to prokaryotic 2-hydroxychromene-2-carboxylate isomerases. Biochem J 2004, 379(Pt3):541-552.

33. Porter D, Weremowicz S, Chin K, Seth P, Keshaviah A, Lahti-Domenici J, Bae YK, Monitto CL, Merlos-Suarez A, Chan J, Hulette CM, Richardson A, Morton CC, Marks J, Duyao M, Hruban R, Gabrielson E, Gelman R, Polyak K: A neural survival factor is a candidate oncogene in breast cancer. Proc Natl Acad Sci U S A 2003, 100:10931-10936.

34. Burwinkel B, Rootwelt T, Kvittingen EA, Chakraborty PK, Kilimann MW: Severe phenotype of phosphorylase kinase-deficient liver glycogenosis with mutations in PHKG2 gene. Pediatr Res 2003, 54:834-839.

35. Chooniedass-Kothari S, Vincett D, Yan Y, Cooper C, Hamedani MK, Myal Y, Leygue $\mathrm{E}$ : The protein encoded by the functional steroid receptor RNA 
activator is a new modulator of ER alpha transcriptional activity. FEBS Lett 2010, 584:1174-1180.

36. Cancer Genome Atlas Network: Comprehensive molecular portraits of human breast tumours. Nature 2012, 490:61-70.

37. Ijichi N, Shigekawa T, Ikeda K, Horie-Inoue K, Shimizu C, Saji S, Aogi K, Tsuda H, Osaki A, Saeki T, Inoue S, Inoue S: Association of double-positive FOXA1 and FOXP1 immunoreactivities with favorable prognosis of tamoxifen-treated breast cancer patients. Horm Cancer 2012, 3:147-159.

38. Hahn WC, Weinberg RA: Rules for making human tumor cells. N Engl J Med 2002, 347:1593-1603.

39. Rubin AF, Green P: Mutation patterns in cancer genomes. Proc Natl Acad Sci U S A 2009, 106(51):21766-21870.

40. Vogelstein B, Papadopoulos N, Velculescu VE, Zhou S, Diaz LA Jr, Kinzler KW: Cancer genome landscapes. Science 2013, 339(6127):1546-1558.

41. Garraway LA, Lander ES: Lessons from the cancer genome. Cell 2013, 153(1):17-37.

42. Shlien A, Tabori U, Marshall CR, Pienkowska M, Feuk L, Novokmet A, Nanda S, Druker H, Scherer SW, Malkin D: Excessive genomic DNA copy number variation in the Li-Fraumeni cancer predisposition syndrome. Proc Natl Acad Sci U S A 2008, 105:11264-11269.

43. MacArthur DG, Balasubramanian S, Frankish A, Huang N, Morris J, Walter K, Jostins L, Habegger L, Pickrell JK, Montgomery SB, Albers CA, Zhang ZD, Conrad DF, Lunter G, Zheng H, Ayub Q, DePristo MA, Banks E, Hu M, Handsaker RE, Rosenfeld JA, Fromer M, Jin M, Mu XJ, Khurana E, Ye K, Kay M, Saunders Gl, Suner MM, Hunt T: A systematic survey of loss-of-function variants in human protein-coding genes. Science 2012, 335(6070):823-828.

doi:10.1186/1471-2407-14-342

Cite this article as: Xiao et al:: Genome instability in blood cells of a BRCA1+ breast cancer family. BMC Cancer 2014 14:342.

\section{Submit your next manuscript to BioMed Central and take full advantage of:}

- Convenient online submission

- Thorough peer review

- No space constraints or color figure charges

- Immediate publication on acceptance

- Inclusion in PubMed, CAS, Scopus and Google Scholar

- Research which is freely available for redistribution 